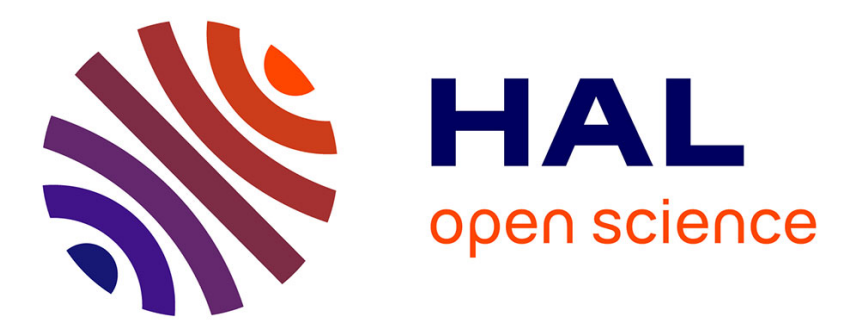

\title{
On the relevance of Continuum Damage Mechanics as applied to the Mullins effect in elastomers
}

Grégory Chagnon, Erwan Verron, Laurent Gornet, Gilles Marckmann, Pierre

Charrier

\section{- To cite this version:}

Grégory Chagnon, Erwan Verron, Laurent Gornet, Gilles Marckmann, Pierre Charrier. On the relevance of Continuum Damage Mechanics as applied to the Mullins effect in elastomers. Journal of the Mechanics and Physics of Solids, 2004, 52 (7), pp.1627-1650. 10.1016/j.jmps.2003.12.006 . hal01007152

\section{HAL Id: hal-01007152 \\ https://hal.science/hal-01007152}

Submitted on 7 Oct 2016

HAL is a multi-disciplinary open access archive for the deposit and dissemination of scientific research documents, whether they are published or not. The documents may come from teaching and research institutions in France or abroad, or from public or private research centers.
L'archive ouverte pluridisciplinaire HAL, est destinée au dépôt et à la diffusion de documents scientifiques de niveau recherche, publiés ou non, émanant des établissements d'enseignement et de recherche français ou étrangers, des laboratoires publics ou privés. 


\title{
On the relevance of Continuum Damage Mechanics as applied to the Mullins effect in elastomers
}

\author{
G. Chagnon ${ }^{\mathrm{a}}$, E. Verron ${ }^{\mathrm{a} ;}{ }^{\mathrm{Q}}$, L. Gornet ${ }^{\mathrm{a}}, \mathrm{G}$. Marckmann ${ }^{\mathrm{a}}$, \\ P. Charrier ${ }^{\mathrm{b}}$ \\ aLaboratoire de Mécanique et Matériaux, École Centrale de Nantes, BP 92101, \\ 44321 Nantes cedex 3, France \\ ${ }^{\mathrm{b}}$ Groupe Trelleborg, Société Modyn, Service Recherche et Innovations, Zone Industrielle Nantes \\ Carquefou, BP 419, 44474 Carquefou cedex, France
}

\begin{abstract}
The present paper reports and rationalizes the use of Continuum Damage Mechanics (CDM) to describe the Mullins effect in elastomers. Thermodynamics of rubber-like hyperelastic materials with isotropic damage is considered. Since it is demonstrated that stress-softening is not strictly speaking a damage phenomenon, the limitations of the CDM approach are highlighted. Moreover, connections with two-network-based constitutive models proposed by other authors are exhibited through the choice of both the damage criterion and the measure of deformation. Experimental data are used to establish the evolution equation of the stress-softening variable, and the choice of the maximum deformation endured previously by the material as the damage criterion is revealed as questionable. Nevertheless, the present model agrees qualitatively well with experiments except to reproduce the strain-hardening phenomenon that takes place as reloading paths rejoin the primary loading path. Finally, the numerical implementation highlights the influence of loading paths on material response and thereby demonstrates the importance of considering the Mullins effect in industrial design.
\end{abstract}

Keywords: Rubber material; Stress-softening; Finite strain; Constitutive equation; Continuum damage mechanics

\footnotetext{
${ }^{\otimes}$ Corresponding author. Tel.: +33-2-40-37-25-14; fax: +33-2-40-37-25-73.

E-mail address: erwan.verron@ec-nantes.fr(E. Verron).
} 


\section{Introduction}

Both natural rubber and synthetic elastomers are widely used in engineering applications, such as tyres, engine mounts or bump stoppers. Due to the growing importance of numerical simulation in the design process of elastomeric parts, a well-established modelling of the material behaviour is an essential prerequisite for the development of new products. It is well known that rubber-like materials exhibit a strongly non-linear behaviour characterized by large strain and a non-linear stress-strain response under static conditions. Moreover, this behaviour is time-dependent as demonstrated by relaxation and creep experiments. Finally, under cyclic loading conditions, both hysteresis and stress-softening phenomena are observed. The hysteretic behaviour may be related to viscoelasticity (Bergström and Boyce, 1998) or viscoplasticity (Lion, 1996), and is characterized by different loading and unloading paths of response during a cycle. The stress-softening phenomenon, also called the Mullins effect, is characterized by an important loss of stiffness during the first cycles of fatigue experiments. More precisely, the Mullins effect is defined as a strain-induced stress-softening of the material that takes place quasi-exclusively after the first loading path of loading cycles (for a detailed description of the Mullins effect, the reader can refer to Drozdov and Dorfmann, 2001).

There is no unanimous microscopic explanation for the stress-softening of elastomers (Marckmann et al., 2002). Pioneering works focused on the experimental description of the phenomenon, and on the development of physically motivated one-dimensional constitutive models (seeMullins (1969) and the references therein). For the last decade, the interest of researchers in the development of constitutive equations involving the Mullins effect has grown. Here, emphasis is laid on three-dimensional models that can be implemented in finite element software and used for engineering applications. Most of the models consider that the Mullins effect takes place during the first cycle of oading and that it only depends on the maximum strain endured previously by the material. These prerequisites are quite acceptable with regard to experimental observations. As the physical explanations of the phenomenon are not well established, a majority of studies propose phenomenological constitutive equations. Two different initial concepts are derived. First, many authors use the thermodynamical framework of Continuum Damage Mechanics (CDM). CDM was first introduced by Kachanov (1958), and the general theory was derived later blyemaitre and Chaboche (1985) for metallic materials. This theory is a particular application of the most general framework of thermodynamics with internal variablesMaugin, 1999). It was successfully applied to different classes of materials such as metals, composites and concrete (see for example Voyiadjis et al., 1998). Recently, CDM was extended to the case of elastomers in order to simulate the Mullins effect. The first paper on the subject is due to Gurtin and Francis (1981) who proposed a one-dimensional hyperelastic damage model for solid propellants. Later, the general three-dimensional case was studied by Simo (1987) who derived a large strain viscoelastic constitutive equation with damage for rubber-like materials. This model was improved by introducing microscopic concepts Govindjee and Simo, 1991, 1992. More recently, several authors developed phenomenological hyperelastic models with damage to describe the Mullins effect in 
engineering applications Qe Souza Neto et al., 1994 Miehe, 1995; Miehe and Keck, 2000). The second approach used to develop constitutive equations for the Mullins effect is based on the two-network theory of Green and Tobolsky (1946) and on the two-phase model ofMullins and Tobin (1957). Both concepts postulate that the polymer network evolves under deformation; they consider that parts of the network are broken and others are reformed during loading. SWVineman and Rajagopal (1990) and Rajagopal and Wineman (1992) proposed a general theory for materials under strain induced micro-structural changes. Their approach is successfully applied to the Mullins effect by Wineman and Huntley (1994), and Huntley et al. (1996, 1997). Authors considered that the stress should be corrected by a scalar reformation function that depends on a given measure of deformation expressed as a scalar function of the principal invariants of the left Cauchy-Green tensor. SimilarlyBeatty and Krishnaswamy (2000) derived a constitutive equation that generalized the previous works of Johnson and Beatty (1993a, b, 1995)on particular deformation states. In this work, the two-phase theory ofMullins and Tobin (1957) is considered, and the transformation of hard regions into soft regions is entirely controlled by a stress-softening function that depends on the maximum strain previously endured by the material. This function corrects the strain-energy function under loading; a different form of it was recently proposed byEl ías-Zúñiga and Beatty (2002). Last, let us mention the recent work oĐgden and Roxburgh (1999); their approach differs from the aforementioned theories because the stress-softening function (that depends on the maximum strain energy endured by the material) is activated only on unloading paths. This theory greatly simplifies the identification of material parameters, but it does not respect the physical phenomenon of network changes during loading. Finally, it should be noticed that the constitutive equations based on network evolution are more specific to polymers than CDM, but the corresponding thermodynamical framework is not as well-defined. Nevertheless, it appears that the constitutive equations obtained by the two approaches, i.e. CDM and network evolution, are close, as shown in the next section.

The aim of the present paper is to demonstrate that Continuum Damage Mechanics can be an efficient tool to model the Mullins effect in rubbers, if it is carefully employed. As most of the phenomenological models developed for the Mullins effect are based on this theory, it is of great importance to rationalize its use and to exhibit its advantages and limitations in this context. Our work is restricted to hyperelasticity with isotropic damage. In Section2, the thermodynamical framework is derived. The emphasis is laid on the restrictions of CDM as applied to the Mullins effect with regard to physical phenomena and on the choice of the damage criterion. The equivalence of CDM and two-network or two-phase approaches is demonstrated. Sections devoted to the experimental part of the work. Experimental data are used to construct the evolution equation of the stress-softening variable and to exhibit some limitations of this approach. The identification of material parameters leads to good qualitative agreement for both uniaxial tensile and simple shear loading conditions. The numerical part of the work is presented in Sectiort. Both plane stress and three-dimensional implementations are considered, and some examples are presented in order to highlight the influence of the loading path on material behaviour. Finally, concluding remarks are given in Section 5 . 


\section{Thermodynamics of rubber-like materials with damage}

2.1. Preliminary remarks on the use of damage mechanics to describe the Mullins effect

As mentioned in the introduction, the Mullins effect in elastomers is often modelled using the CDM. Before examining in detail the isotropic CDM as applied to non-linear elastic materials under large strain, let us first make some remarks on this subject.

Originally, damage mechanics was based on the definition of effective stress. This theory considers that, under loading, the material surface on which internal forces apply is decreasing because of the emergence of micro-defects and micro-voids. Taking into account these physical considerations, three consequences are induced for the damage evolution:

1. the damage cannot decrease during material life because micro-defects and microvoids cannot disappear,

2. the damage evolution differs depending on loading conditions: under tension both micro-defects and voids appear, and under compression only micro-defects are activated because micro-voids are closed,

3. the damage evolves until occurrence of a sufficiently large crack (or rupture), because the active surface tends to a non-zero threshold value determined experimentally (or to zero).

Nevertheless, in the case of the Mullins effect in elastomers, these three assertions can be refuted:

1. it was proved experimentally that the Mullins effect recovers with time and that this recovery is highly accelerated by annealing Mullins, 1969). So, the damage may decrease if the whole life of the material is considered,

2. the damage evolution does not differ under different deformation modes: some authors observed the occurrence of stress-softening under compressive conditions (Bergström and Boyce, 1998),

3. it is mainly recognized that rupture in rubber parts is not directly related to stresssoftening. Therefore, high values of the damage parameter should not be considered as a criterion for rupture or occurrence of cracks.

More precisely, the Mullins effect is not a damage but a stress-softening phenomenon. It is due to the rearrangement of the polymer network under deformation when some links between chains (entanglements), or between chains and reinforcement particles (for example carbon black) are broken downBueche, 1960, 1961). Considering these remarks, it should be concluded that CDM can be used to model the Mullins effect in elastomers with some restrictions and care.

\subsection{Derivation of the constitutive equation}

Consider an isotropic, homogeneous and incompressible rubber-like material. This material is considered hyperelastic and subject to isotropic damage in order to describe 
the Mullins effect. Consequently, it is defined by the existence of a strain energy function, that depends on the deformation gradientand on a scalar damage variable d. This variable characterizes the elastic stress-softening of the material. Taking into account the objectivity requirement, the isotropy and the incompressibility, the strain energy function can be written as

$$
W=W\left(I_{1} ; I_{2} ; d\right) \text {; }
$$

where $I_{1}$ and $I_{2}$ are the two first principal invariants of the left Cauchy-Green tensor $B=F^{\top} F$ defined by

$$
\mathrm{I}_{1}=\operatorname{tr} \mathrm{B} \quad \text { and } \quad \mathrm{I}_{2}=\frac{1}{2}\left(I_{1}^{2}-\operatorname{tr}\left(\mathbf{B}^{2}\right)\right) \text {. }
$$

The third invariant $I_{3}=\operatorname{det} \mathbf{B}$ is equal to 1 due to the incompressibility assumption.

Considering now that the effective Cauchy stress $\boldsymbol{\sigma}_{0}$, which acts on the damaged material, is related to the applied Cauchy stress $\sigma$ by Lemaitre and Chaboche (1985)

$$
\boldsymbol{\sigma}_{0}=\frac{\boldsymbol{\sigma}}{1-d}
$$

then, the strain energy function Eq. (1) can be considered as the product of the surface reducing parameter $1-d$ and the strain energy function of the virgin undamaged material, denoted $W_{0}$ :

$$
W\left(I_{1}, I_{2}, d\right)=(1-d) W_{0}\left(I_{1}, I_{2}\right)
$$

In order to establish the laws of state, the Clausius-Duhem inequality has to be considered. It yields (Miehe, 1995)

$$
\mathscr{D} \stackrel{\text { def }}{=} \boldsymbol{\sigma}: \mathbf{D}-\dot{W} \geqslant 0
$$

in which $\mathscr{D}$ stands for the internal dissipation and $\mathbf{D}$ is the rate of deformation tensor that satisfies $\operatorname{tr} \mathbf{D}=0$ due to incompressibility. After some algebraic manipulations, Eq. (5) can be cast into the following form:

$$
\mathscr{D}=\left(\boldsymbol{\sigma}-2 \mathbf{B} \frac{\partial W}{\partial \mathbf{B}}\right): \mathbf{D}-\frac{\partial W}{\partial d} \dot{d} \geqslant 0
$$

with $\operatorname{tr} \mathbf{D}=0$. Thus, examining the two terms of this inequality, both the stress-strain relationship and the dissipation can be established:

$$
\boldsymbol{\sigma}=-p \mathbf{I}+(1-d) 2 \mathbf{B} \frac{\partial W_{0}}{\partial \mathbf{B}} \quad \text { and } \quad \mathscr{D}=W_{0} \dot{d} \geqslant 0,
$$

where $p \mathbf{I}$ is an arbitrary spherical tensor which expresses that only stress differences are defined for incompressible materials. Indeed, the hydrostatic pressure $p$ cannot be obtained using the stress-strain relationships but is deduced from the governing equations of the problem. Eq. $(7)_{1}$ is the constitutive equation that relates the current damaged stress to the damage parameter, the strain energy function of the virgin material and the current strain state. Using that $W_{0}$ only depends on the two first principal invariants 
of $B$, it becomes

$$
\boldsymbol{\sigma}=-\mathrm{pl}+(1-\mathrm{d})\left[2\left(\frac{@ \mathrm{~W}}{\partial I_{1}}+I_{1} \frac{\partial W_{0}}{\partial I_{2}}\right) \mathbf{B}-2 \frac{\partial W_{0}}{\partial I_{2}} \mathbf{B}^{2}\right]
$$

in which the functions $\partial W_{0} / \partial I_{1}$ and $\partial W_{0} / \partial I_{2}$ are the material parameters. Eq. $(7)_{2}$ shows that the damage process is dissipative and that the thermodynamic force associated with the damage variable $d$ is

$$
Y=\frac{\partial W}{\partial d}=-W_{0}
$$

Next, in order to completely define the damaged hyperelastic constitutive equation, the evolution equation for the damage variable $d$ has to be considered. As mentioned in the introduction, it is mainly recognized that the stress-softening in the material exclusively depends on the maximum deformation endured by the material during its history (Mullins, 1969). Therefore, a measure of the deformation state and its maximum should be defined. This measure depends on the deformation history and is denoted $\alpha$. The choice of $\alpha$ will be discussed later. Its maximum is

$$
\bar{\alpha}(t)=\max _{\tau \in]-\infty, t]} \alpha(\mathbf{B}(\tau)) .
$$

In the following, notations proposed by Simo and Hughes (1998) are employed. Thus, considering previous definitions, the damage criterion provides a sub-region of the deformation space given by (Miehe, 1995; Simo and Hughes, 1998):

$$
\phi(\alpha(\mathbf{B}(t)), \bar{\alpha}(t)) \stackrel{\text { def }}{=} \alpha(\mathbf{B}(t))-\bar{\alpha}(t) \leqslant 0 .
$$

The corresponding damage surface and its normal are, respectively

$$
\phi=0 \quad \text { and } \quad \mathbf{n} \stackrel{\text { def }}{=} \frac{\partial \phi}{\partial \mathbf{B}} .
$$

So, for a particular deformation state, four physical situations and their mathematical counterparts are possible:

(i) the current deformation state is not maximum $(\alpha(t) \neq \bar{\alpha}(t))$, then the inequality $\phi<0$ is satisfied (no damage evolution),

(ii) the current deformation state is maximum $(\phi=0)$ and is in an unloading direction from a damage state $(\mathbf{n}: \dot{\mathbf{B}}<0)$,

(iii) the current deformation state is maximum $(\phi=0)$ and is in a neutral direction from a damage state $(\mathbf{n}: \dot{\mathbf{B}}=0)$,

(iv) the current deformation state is maximum $(\phi=0)$ and is in a loading direction from a damage state $(\mathbf{n}: \dot{\mathbf{B}}>0)$.

The three first cases (i)-(iii) correspond with physical situations in which the damage should not evolve, and the fourth one (iv) represents the case in which the maximum of the deformation in the material life changes, so that the damage parameter increases. Consequently, an irreversible evolution equation for the damage can be proposed as 
follows:

$$
\dot{\mathrm{d}}= \begin{cases}\mathrm{h}(\alpha, d) \dot{\alpha} & \text { if } \phi=0 \text { and } \mathrm{n}: \dot{\mathrm{B}} i \quad 0 ; \\ 0 & \text { otherwise }\end{cases}
$$

where the functionh characterizes the damage evolution. In the case of the Mullins effect, the dependence of $h$ on the current value of the damage parametcis classically not considered, so the first case of Eq. (13) reduces to

$$
\dot{\mathrm{d}}=\mathrm{h}(\alpha) \dot{\alpha} \quad \text { with } \alpha=\bar{\alpha} \text { and } \dot{\alpha}>0 \text { : }
$$

As the damage is an irreversible non-decreasing variable, i.e $i \quad 0$, the functionh is a positive function on $] \varphi+\infty$ ). Moreover, considering thath is sufficiently smooth (that is ensured by the physics of stress-softening) and that the damage in the natural state is equal to zero, Eq. (14) can be integrated to give

$$
\mathrm{d}=\tilde{\mathrm{d}}(\bar{\alpha})=\int_{0}^{\bar{\alpha}} \mathrm{h}(\alpha) \mathrm{d} \alpha
$$

Consequently, the stress-strain relationship Eq.80 simply becomes

$$
\boldsymbol{\sigma}=-\mathrm{pl}+(1-\tilde{\mathrm{d}}(\bar{\alpha}))\left[2\left(\frac{@ W_{6}}{\partial I_{1}}+I_{1} \frac{\partial W_{0}}{\partial I_{2}}\right) \mathbf{B}-2 \frac{\partial W_{0}}{\partial I_{2}} \mathbf{B}^{2}\right] .
$$

It may be noted that this equation is similar to constitutive equations proposed by others for the Mullins effect (Gurtin and Francis, 1981; De Souza Neto et al., 1994; Miehe, 1995; Beatty and Krishnaswamy, 2000; Elías-Zúñiga and Beatty, 2002), even if some of these theories were not established using the CDM theory.

\subsection{Discussion on the scalar measure of deformation state}

The last problem to solve is the choice of the measure of deformation state. In the general theory of thermodynamics of irreversible processes, the evolution equation of an internal variable has to be written in terms of the thermodynamical force associated with this variable, because it avoids considering constitutive equations that do not satisfy the second law of thermodynamics. In the present case, it means that the measure $\alpha$ should be chosen as a function of $Y$, the thermodynamical force associated with $d$, that is equal to $-W_{0}$. In fact, $W_{0}$ can be seen as a measure of the deformation state and this choice is consistent with data provided in experiments. The first authors who proposed to apply CDM to the modelling of the Mullins effect adopted this approach, because they decided to apply the CDM theory without deviation (Simo, 1987; De Souza Neto et al., 1994; Miehe, 1995). This choice exhibits one major problem: the form of the strain energy function should be postulated before derivation of the whole model. Moreover, due to physical considerations, it seems to be more appropriate to formulate explicitly the damage evolution equation in terms of the deformation state. That was proposed, in the context of CDM, by Govindjee and Simo (1991, 1992), and later by Miehe and Keck (2000). Note that this approach can be related to the use of the 
two-network theory ofGreen and Tobolsky (1946) or the two-phase theory ofMullins and Tobin (1957) to model the Mullins effect. Indeed, in their papers, Wineman and Rajagopal (1990), Johnson and Beatty (1993a) and Beatty and Krishnaswamy (2000) consider that the Mullins effect is due to a change in the micro-structure of the network. The evolution of the network is described by a reformation function that depends on the maximum strain state endured by the material during its life. In the aforementioned papers, the deformation state is measured through a scalar function which depends on $I_{1}$ and $I_{2}$. As proposed by Beatty and Krishnaswamy, this function can be seen as a damage parameter (see footnote 5 iBeatty and Krishnaswamy, 2000).

First the basic application of the CDM theory is examined. Let $(B)=f\left(W_{0}(B)\right)$ be the measure of the deformation state in terms of the thermo-dynamical force associated with $d$. In the following, the simplified notation $f\left(W_{0}\right)$ is adopted. Thus, even if $f$ has not to be a norm, it can be reasonably stated that:

- $f$ is defined and differentiable on $[0 ;+\infty)$,

- $f(0)=0$, i.e. the measure of the undeformed state is null,

- $f$ is an increasing function on $[0+\infty)$.

Consequently, $f$ is a positive increasing function on $]$, $0+\infty)$. Using this measure of the deformation state, the material isotropy and the chain-rule differentiation, Eq. (14) becomes

$$
\dot{d}=h\left(f\left(W_{0}\right)\right) f ; w_{0}\left(W_{0} i_{1} \dot{l}_{1}+W_{0} i_{2} \dot{l}_{2}\right):
$$

Taking into account the E-inequalities proposed byruesdell and Noll (1965, p. 158):

$$
W_{0 i i_{1}} i \quad 0 \text { and } W_{0 ; i_{2}} i \quad 0
$$

the previous Eq. (17) can be written as

$$
\dot{\mathrm{d}}=\beta_{1}^{\mathrm{f}} \dot{\mathrm{l}}_{1}+\beta_{2}^{\mathrm{f}} \dot{\mathrm{l}}_{2} ;
$$

where $\left(\beta_{\mathrm{i}}^{\mathrm{f}}\right)_{\mathrm{i}=1 ; 2}$ are functions of $\mathrm{I}_{1}$ and $\mathrm{I}_{2}$, and satisfy

$$
\left.\left.\beta_{1}^{\mathrm{f}} i \quad 0 \text { and } \beta_{2}^{\mathrm{f}} i \quad 0 \text { on }\right] 0 ;+\infty\right) \text { : }
$$

The classical CDM approach being derived, the second method that consists in directly expressing the scalar measure of the deformation state in terms of the left Cauchy-Green tensor is examined. For isotropic and incompressible materials, the measure $\alpha(B)$ only depends on the two first principal invariants:

$$
\alpha(B)=g\left(I_{1} ; I_{2}\right):
$$

Following exactly the same method as above, Eq.1(4) gives

$$
\dot{d}=h\left(g\left(l_{1} ; l_{2}\right)\right)\left(g_{i i_{1}} \dot{l}_{1}+g_{i_{2}} l_{2}\right) \text { : }
$$

Comparing this equation with Eqs. 19) and (20), and recalling the properties ofh, the properties thatg should satisfy can be easily established:

- $g$ is defined on $[3 ;+\infty) \times[3 ;+\infty)$,

- $g$ is differentiable in both variables $\mathrm{I}_{1}$ and $\mathrm{I}_{2}$ on $[3 ;+\infty) \times[3 ;+\infty)$, 
- $g_{i_{1}} i \quad 0$ and $g_{i_{2}} i \quad 0$,

- the choice $g\left(I_{1}=3 ; I_{2}=3\right)=0$, that ensures that the measure is null for the virgin unloaded state, is recommended.

\section{Experiments and determination of material parameters}

\subsection{Experimental results}

The material used for the experimental part of this work is a carbon black filled natural rubber. The percentage of fillers is about $30 \%$. This material was furnished by the Trelleborg group and all experiments were conducted in its French Research Department.

In order to determine material parameters, that are the virgin strain energy function $W_{0}$ and the evolution equation of the damage variake $(\hat{\alpha} \bar{\alpha})$, cyclic uniaxial tensile and simple shear tests were performed. The uniaxial tensile experiments were conducted on flat coupon specimens and the simple shear specimens are four blocks pieces. All experiments were performed under enforced displacement conditions. For several strain levels from $25 \%$ up to $500 \%$ for uniaxial tensile and from $20 \%$ up to $480 \%$ for simple shear experiments, specimens underwent five cycles at a constant strain rate set to 4:167 $\mathrm{s}^{-1}$. An example of experimental data is depicted in Figh.(a) for uniaxial tensile and (b) for simple shear tests. In this figure, experimental stresses are normalized by the maximum stress measured in uniaxial tension. In the case of uniaxial tension, it is clearly shown that the Mullins effect occurs almost exclusively during the first loading cycle, and all secondary cycles, i.e. second, third, fourth and fifth cycles, are close. Thus, only stress-softening taking place between the first and the second cycles is considered. It can be assimilated to the term "discontinuous damage" previously introduced byMiehe (1995). It is to be noted that reloading paths do not rejoin the primary loading curve at the maximum strain. In fact, as samples are unloaded to zero displacement, they remain in a deformed configuration due to the time-dependent behaviour of the material (creep phenomenon). This is especially the case for flat coupon specimens that buckle during unloading. Consequently the next reloading stretch level should be higher than the first loading stretch level to rejoin the first path (Johnson and Beatty, 1993a).

As in most of the studies concerned with the Mullins effect, the present paper only deals with the loss of stiffness of the material. Therefore, the stress-softening behaviour should be separated from other inelastic phenomena such as hysteresis and relaxation. Concerning the hysteresis phenomenon, unloading paths of cycles are not considered and it is assumed that the equilibrium paths are the loading paths. In fact, static paths that correspond to the thermodynamic equilibrium of the material are situated within hysteresis cycles, i.e. between loading and unloading paths, but their exact positions are not well-established Bergström and Boyce, 1998). Nevertheless, the stress-softening model can be develop for loading paths, then in further works the results obtained here would be adapted to static paths without major difficulties. Moreover, the relaxation phenomenon can be eliminated by correcting the reference dimensions of samples in 

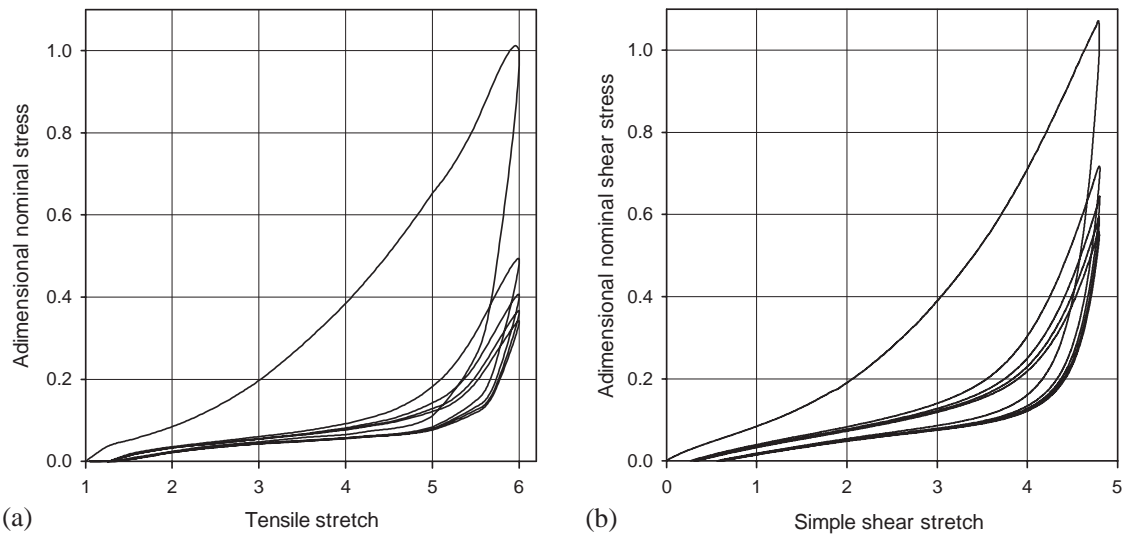

Fig. 1. Experimental results: (a) uniaxial tensile and (b) simple shear.
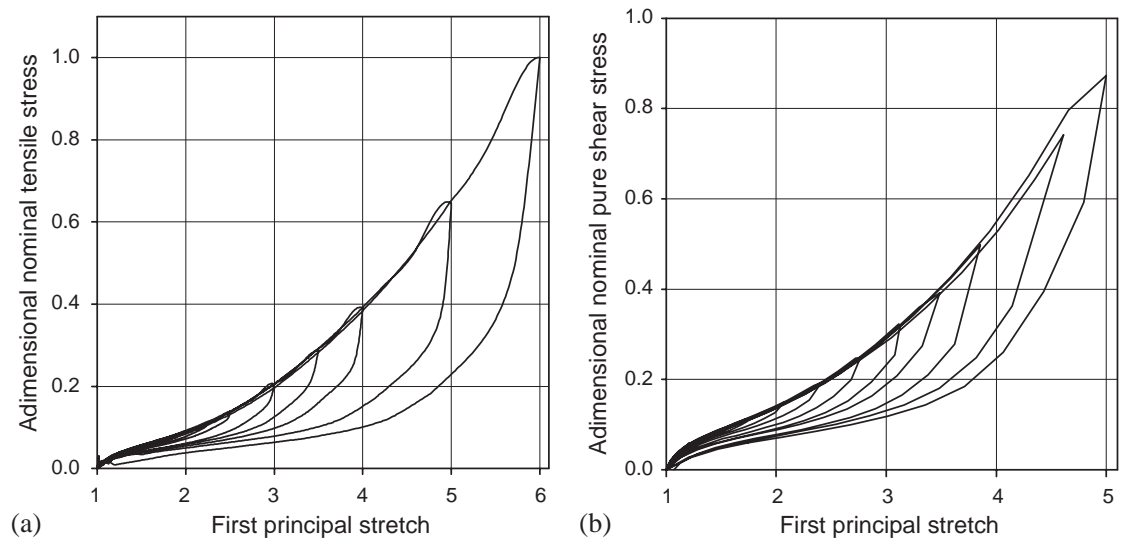

Fig. 2. Corrected data: (a) uniaxial tensile and (b) pure shear.

the unloaded configuration for the next loadings. Practically, experimental results are corrected in three steps: (i) only loading paths are considered, (ii) reloading paths are shifted to zero to eliminate the permanent set and (iii) they are extended to rejoin the first loading path.

Finally, note that the analysis of simple shear experiments leads to some difficulties for the determination of material parameters, because principal directions of strain do not remain constant in time. Here, in order to simplify the analysis, simple shear experimental data are transformed into pure shear data, as proposed by Charlton et al. (1994). Note that pure shear data could have been obtained with an appropriate experimental apparatus (Rivlin, 1948) but it is practically easier to consider simple shear experiments. The corrected experimental data are presented in Figs. 2(a) and (b) for uniaxial tensile and pure shear results, respectively. 


\subsection{Determination of material parameters}

As shown in Section 2, the general framework of hyperelasticity with damage necessitates the determination of two material functions: the virgin strain energy function $W_{0}$ and the evolution equation of the stress-softening variable (Eqsi5l and (21)). Forms of these two functions are established in the next paragraphs.

\subsubsection{Strain energy function}

Several forms for the strain energy function of rubber-like materials have been proposed since 1940. Two different kinds of models can be identified. The first kind is based on experimental observations and phenomenological considerations. The corresponding models are both mathematically simple and quite efficient (Mooney, 1940; Rivlin and Saunders, 1951; Gent and Thomas, 1958 Hart-Smith, 1966; Ogden, 1972 Yeoh, 1990). The second group of models were developed by considering the physics of the elastomer network. First, conformations of a single polymer chain are determined; then, the behaviour of the whole network is derived using statistical developments. For small and moderate strains, the neo-hookeanreloar, 1944) and the phantom James and Guth, 1947 models can be mentioned. For large strains, the stretching limit of chains is taken into account and non-Gaussian statistics are employdklłhn and Grün, 1942; James and Guth, 1943 Treloar and Riding, 1979; Arruda and Boyce, 1993; $\mathrm{Wu}$ and van der Giessen, 1993. The use of these models reduces the number of relevant material parameters because of their physical foundations, but their mathematical derivations are more complicated than those of phenomenological constitutive equations. For a complete review of the constitutive models for rubber elasticity, the reader can refer to Boyce and Arruda (2000).

It has to be mentioned that the present approach can be easily applied to every form of the strain energy function. In the present study, the emphasis is laid on the stress-softening phenomenon, so that a simple phenomenological strain energy function is chosen. Then, recalling that in the case of ncompressible materiaVs ${ }_{0}$ is a function of the two first invariants $I_{1}$ and $I_{2}$, the general expansion ofRivlin (1948) is considered:

$$
W_{0}=\sum_{i ; j} C_{i j}\left(I_{1}-3\right)^{i}\left(I_{2}-3\right)^{j}
$$

in which $C_{\mathrm{ij}}$ are the material parameters. Several phenomenological models were derived by truncating the series Eq. 23). In order to satisfactorily reproduce the large strain response of the material, the Yeoh model is adoptede(bh, 1990, 1993). The corresponding strain energy function is given by

$$
\mathrm{W}_{0}=\sum_{\mathrm{i}=1}^{3} \mathrm{C}_{\mathrm{i} 0}\left(\mathrm{I}_{1}-3\right)^{\mathrm{i}}
$$

where $C_{10}, C_{20}$ and $C_{30}$ are the three material parameters. As this model only depends on the first principal invariant, it is not well-adapted to moderate strain because it does not improve the Gaussian theory. Nevertheless, its mathematical simplicity and ability 
to reproduce the whole behaviour of elastomers are sufficient qualities for the present study.

Using Eqs. (8) and (24), the stress-strain relationship reduces to

$$
\boldsymbol{\sigma}=-\mathrm{pl}+2(1-\tilde{\mathrm{d}}(\bar{\alpha})) \sum_{\mathrm{i}=1}^{3} \mathrm{iC}_{\mathrm{i} 0}\left(\mathrm{I}_{1}-3\right)^{\mathrm{i}-1} \mathrm{~B}:
$$

Hence, the analytical stress-strain equations corresponding to our experiments can be easily derived (see Ogden (1984) for details). First, in the case of uniaxial tensile tests, both the stretch $\lambda$ and the nominal stress $\pi$ in the tensile direction are measured. The corresponding equation is given by

$$
\pi=2(1-\tilde{\mathrm{d}}(\bar{\alpha}))\left(\lambda-\frac{1}{\lambda^{2}}\right) \sum_{i=1}^{3} i C_{i 0}\left(\lambda^{2}+\frac{2}{\lambda}-3\right)^{i-1} .
$$

Second, after transformation of simple shear results into pure shear data, the corresponding relation between the nominal stress and the stretch in the first principal direction, i.e. the one that corresponds to the maximum stretch, reduces to

$$
\pi=2(1-\tilde{d}(\bar{\alpha}))\left(\lambda-\frac{1}{\lambda^{3}}\right) \sum_{i=1}^{3} i C_{i 0}\left(\lambda^{2}+\frac{1}{\lambda^{2}}-1\right)^{i-1} .
$$

\subsubsection{Evolution equation of the damage variable}

Once the virgin strain energy function has been chosen, the form of the damage evolution equation (Eqs. (14) and (15)) has to be established. As detailed in the theoretical part of the paper (Section 2), the present approach focuses on the use of a measure of deformation expressed as a function of the two first invariants of $\mathbf{B}$ (Eq. (21)). As a first approach, we adopt a very simple measure of the deformation state that only depends on $I_{1}$ :

$$
\alpha=\sqrt{I_{1} / 3}-1
$$

in which $\sqrt{I_{1} / 3}$ is the stretch of the diagonal of a material cube. This measure is relevant to the properties exhibited in the theoretical section: it is defined and differentiable on $[3,+\infty) \times[3,+\infty)$, its partial derivative with respect to $I_{1}$ is positive and its partial derivative with respect to $I_{2}$ is equal to zero, and finally the measure is null as $I_{1}=3$ (whatever the value of $I_{2}$ ). Note that the change of deformation measure is obvious, the only problem being the availability of sufficient experimental results to identify the corresponding function.

The damage function $\tilde{d}(\alpha)$ will be now determined using experimental results. The general method followed here is described without reference to the kind of experiments, but results for both uniaxial tensile and pure shear data are presented. First, new notations are defined. Consider experimental results as sketched in Fig. 3. Using reduced experimental data, the stress-strain relationship reduces to a sequence of loading curves at different maximum strains. The corresponding curves can represent either uniaxial tensile and pure shear data. In this figure, the nominal stress $\pi$ is given as a function of the first invariant $I_{1}$. Two types of stress-strain curves can be identified: the primary 


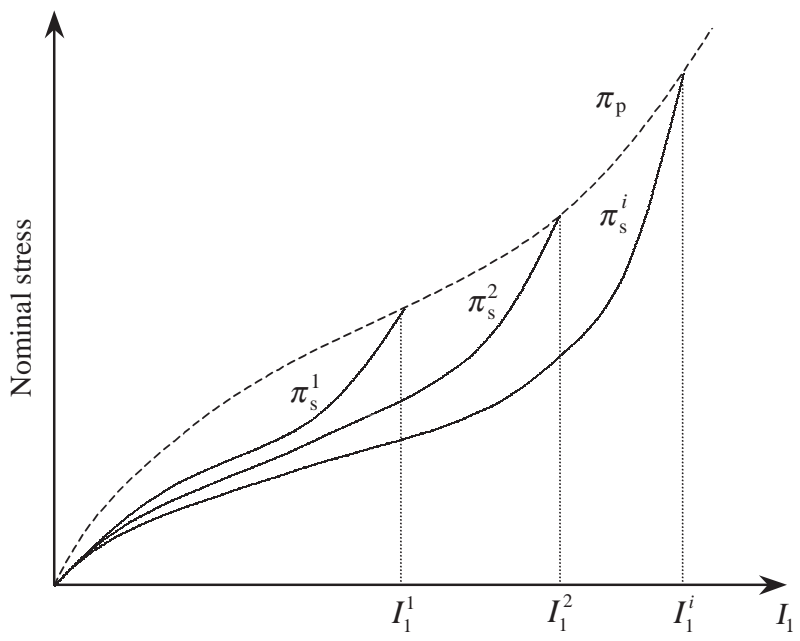

Fig. 3. Notations used for the construction of the evolution equation of the damage variable: (- -) primary loading curve and (-) secondary loading curves.

curve represented by a dashed line and secondary curves represented by solid lines. Depending on the type of curve, the stress-strain relationship differs:

- for the primary curve, the current stress $\pi_{\mathrm{p}}$ is given by

$$
\pi_{\mathrm{p}}=k_{\mathrm{p}} \pi_{0},
$$

where $\pi_{0}$ is the nominal stress of the virgin material and $k_{\mathrm{p}}$ depends on $I_{1}$ :

$$
k_{\mathrm{p}}=1-\tilde{d}(\bar{\alpha}) \quad \text { with } \bar{\alpha}=\sqrt{I_{1} / 3}-1 .
$$

So, on the primary curve $k_{\mathrm{p}}$ is decreasing as $I_{1}$ increases,

- on the $i$ th secondary curve delimited by its intersection at $I_{1}^{i}$ with the primary curve, the current stress $\pi_{\mathrm{s}}^{i}$ can be written as

$$
\pi_{\mathrm{s}}^{i}=k_{\mathrm{s}}^{i} \pi_{0}
$$

where $k_{\mathrm{s}}^{i}$ is a constant parameter given by

$$
k_{\mathrm{s}}^{i}=1-\tilde{d}(\bar{\alpha}) \quad \text { with } \bar{\alpha}=\sqrt{I_{1}^{i} / 3}-1 .
$$

Indeed, on this curve, the current value of $\alpha$ is lower than $\bar{\alpha}$ previously reached on the primary curve for $I_{1}=I_{1}^{i}$.

Consequently, for two different secondary curves numbered $i$ and $j(i<j)$, the corresponding nominal stresses are simply related by a multiplicative factor:

$$
\frac{\pi_{\mathrm{s}}^{j}}{\pi_{\mathrm{s}}^{i}}=\frac{k_{\mathrm{s}}^{j}}{k_{\mathrm{s}}^{i}} \quad \text { on }\left[1, I_{1}^{i}\right] \text {. }
$$




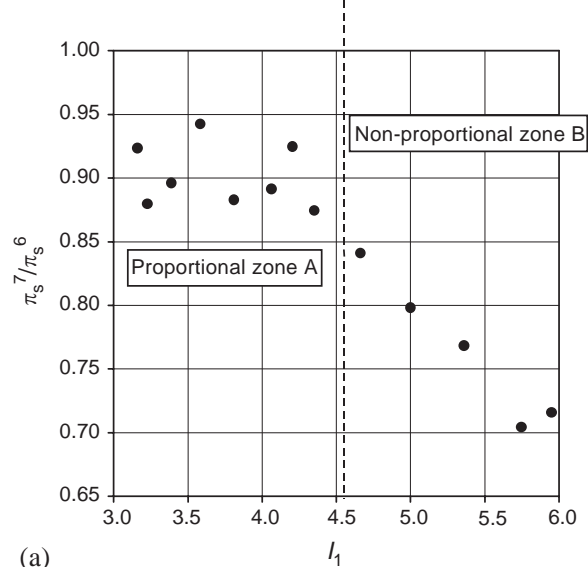

(a)

Fig. 4. Examples of ratio $\pi_{\mathrm{s}}^{i+1} / \pi_{\mathrm{s}}^{i}$ : (a) uniaxial tensile and (b) pure shear.

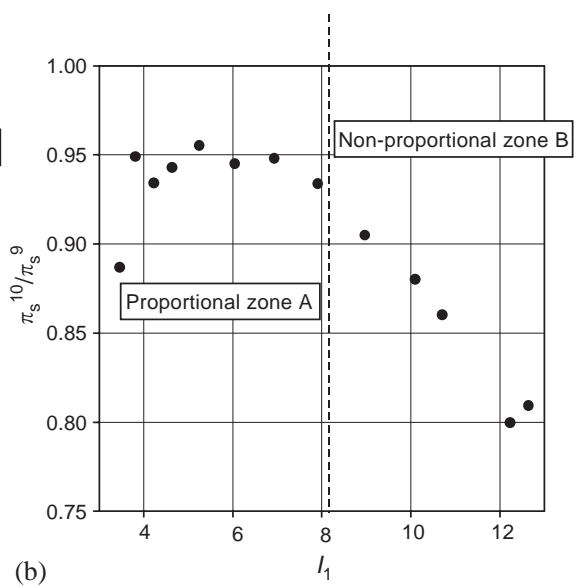

(b) 

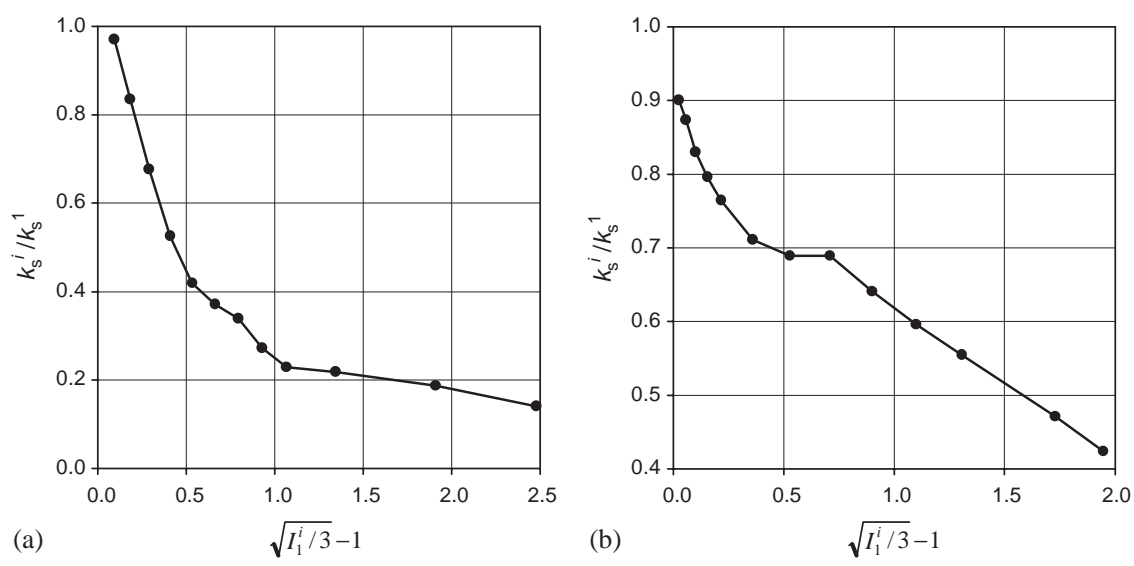

Fig. 5. Evolution of the non-dimensional damage parameter $k_{\mathrm{s}}^{i} / k_{\mathrm{s}}^{1}$ as a function of the measure of deformation $\sqrt{I_{1}^{i} / 3}-1$ : (a) uniaxial tensile and (b) pure shear.

where $a, b$ and $c$ are material parameters. Moreover, considering that in the initial unloaded state the material is undamaged, i.e. $d=0$ and $k=1$ for $I_{1}=3$, then the evolution equation of $k$ reduces to

$$
k=1+b^{\prime}\left\{\exp \left[-c\left(\sqrt{I_{1} / 3}-1\right)\right]-1\right\} .
$$

Recalling that $k=1-d$, the evolution equation of $d$ can be cast into the following form:

$$
\tilde{d}(\bar{\alpha})=d_{\infty}\left[1-\exp \left(-\frac{\bar{\alpha}}{\eta}\right)\right],
$$

where the two new material parameters are the maximum damage $d_{\infty}$ and a scale factor $\eta$. They are related to the previous parameters by

$$
d_{\infty}=b k_{\mathrm{s}}^{1} \quad \text { and } \quad \eta=1 / c .
$$

The form of the evolution equation Eq. (36) is similar to the one proposed phenomenologically by Miehe (1995), except that his model is expressed in terms of the strain energy function: the measure $\alpha$ in Eq. (36) is replaced by $W_{0}$. Finally, note that experimental damage curves for uniaxial tensile and pure shear data are qualitatively similar but their corresponding parameters in Eq. (36) are different (see Figs. $5(\mathrm{a})$ and (b)). This difference is also explained in the next discussion.

\subsubsection{Model limitations and fitting results}

3.2.3.1. Limitations As mentioned above, the continuum damage mechanics as applied to the modelling of the Mullins effect is only effective in the proportional part of the experimental curves (see Fig. 4). This limitation of the present theory is 

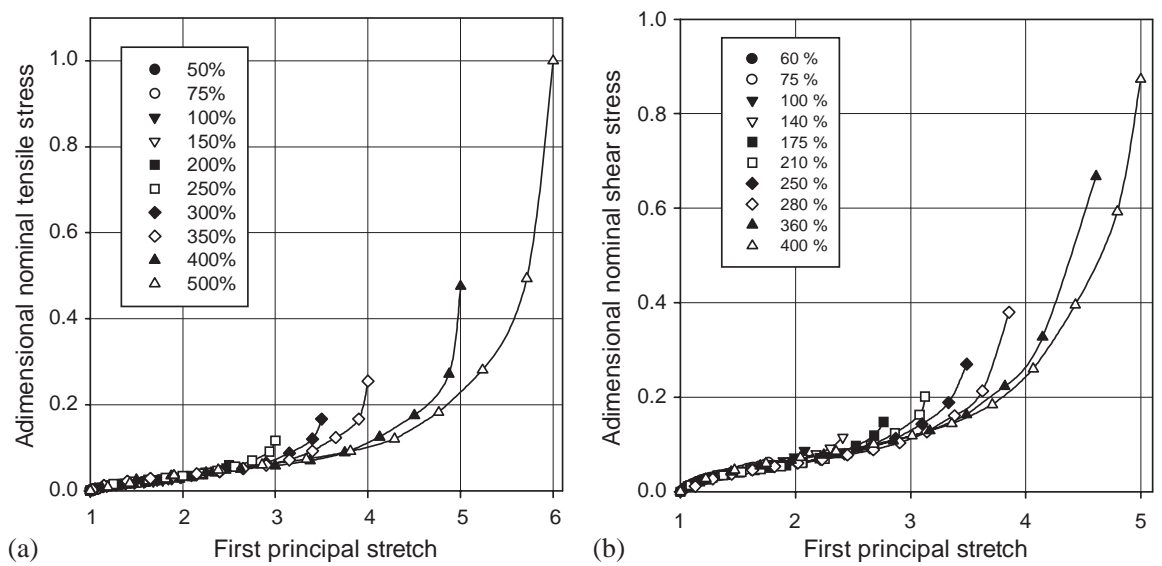

Fig. 6. Superimposition of the secondary loading curves: (a) uniaxial tensile and (b) pure shear. Note that strain-hardening is not satisfactorily reproduced.

highlighted in Fig. 6, in which experimental secondary loading curves were superimposed using the scale factors $k_{\mathrm{s}}^{i}$. It shows that the superimposition is almost perfect until the phenomenon of strain-hardening takes place. This phenomenon occurs as secondary curves rejoin the primary curve and corresponds to the level of stress-softening previously endured by the material. In fact, this limitation is inherent in the choice of the damage mechanism: as the evolution equation of the damage variable is written in terms of a maximum deformation state (using the strain energy or a measure of deformation), theoretical secondary curves are proportional. Physically, this discrepancy can be explained by considering the evolution of the network and the breaking of different links in it, as recently proposed by Marckmann et al. (2002).

The second difficulty evoked in the previous paragraph is the quantitative difference between evolution equations of the stress-softening variable obtained with uniaxial tensile and pure shear experimental data. In our opinion, this difference is a consequence of the choice of the deformation measure. Here, for the sake of simplicity, the measure $\alpha$ only depends on $I_{1}$. In order to reproduce several loading conditions, the influence of $I_{2}$ should be also retained. Nevertheless, it requires a large number of experimental data corresponding with different loading conditions.

Finally, the range of deformation in which the model is applicable should be estimated. Theoretically, the damage $d$ should never be greater than 1 . If the long-term damage parameter $d_{\infty}$ in Eq. (36) is lower than 1 , then $d \leqslant 1$ is satisfied for all deformation states. The constraint $d_{\infty} \leqslant 1$ could be imposed during the identification process. Here, this constraint is not imposed and $d_{\infty}$ is allowed to be greater than 1. In that case, the model remains valid only if $d \leqslant 1$, and this condition can be written as

$$
I_{1} \leqslant 3\left[1+\eta \ln \left(\frac{d_{\infty}}{d_{\infty}-1}\right)\right] .
$$


Table 1

Values of material parameters

\begin{tabular}{ll}
\hline Parameters & Values \\
\hline$C_{10}$ & $3.99 \mathrm{e}^{-2} \mathrm{MPa}$ \\
$C_{20}$ & $-4.05 \mathrm{e}^{-4} \mathrm{MPa}$ \\
$C_{30}$ & $1.31 \mathrm{e}^{-4} \mathrm{MPa}$ \\
$d_{\infty}$ & 1.744 \\
$\eta$ & 3.85 \\
\hline
\end{tabular}

Thus, for finite element applications, the previous inequality should be satisfied in all points of the structure in order to ensure the validity of the results. This is especially the case in parts of the mesh where important local deformation is predicted.

3.2.3.2. Fitting results Five material parameters have to be determined: three for the virgin strain energy, i.e. $C_{10}, C_{20}$ and $C_{30}$, and two for the evolution equation of the damage, i.e. $d_{\infty}$ and $\eta$. Due the use of a measure of deformation instead of the strain energy function as the damage criterion, the evolution equation could be fitted firstly using data reported in Fig. 5; and then the determination of $W_{0}$ reduces to the classical problem of fitting a hyperelastic model using the primary loading curve. Nevertheless, taking into consideration limitations discussed above, it is more efficient to perform a global identification using the stress-strain data for both uniaxial tensile and pure shear tests.

For the identification process, the error between the model and experimental results is calculated simultaneously on all curves. Computations are performed using two different algorithms. First, an optimization program based on a genetic algorithm estimates approximately the material parameters. Second, these parameters are precisely determined with the help of a classical steepest descent algorithm using the genetic algorithm results as initial guess solutions.

The values of material parameters obtained are presented in Table 1 and the identification results are compared with experiments in Figs. 7(a) and (b) for uniaxial tensile and pure shear deformation state, respectively. Theoretical results are globally in good agreement with experiments; so, the model describes successfully the transition between different loading curves in cyclic experiments and exhibits the influence of the maximum strain on the softened behaviour. As anticipated, the present model is not able to satisfactorily predict the behaviour of the material at the intersection of secondary loading curves with the primary curve. Indeed, the high curvature of secondary loading curves due to strain-hardening is not well reproduced. Nevertheless, such results with only five material parameters are good, and the use of the present model in finite element simulations will provide quality results. Finally, considering Eq. (38) and the values of material parameters given in Table 1, the present approach is applicable until $630 \%$ of deformation in uniaxial tension and up to $400 \%$ in equibiaxial tension. In this way, simulation of large strain problems can be considered. 

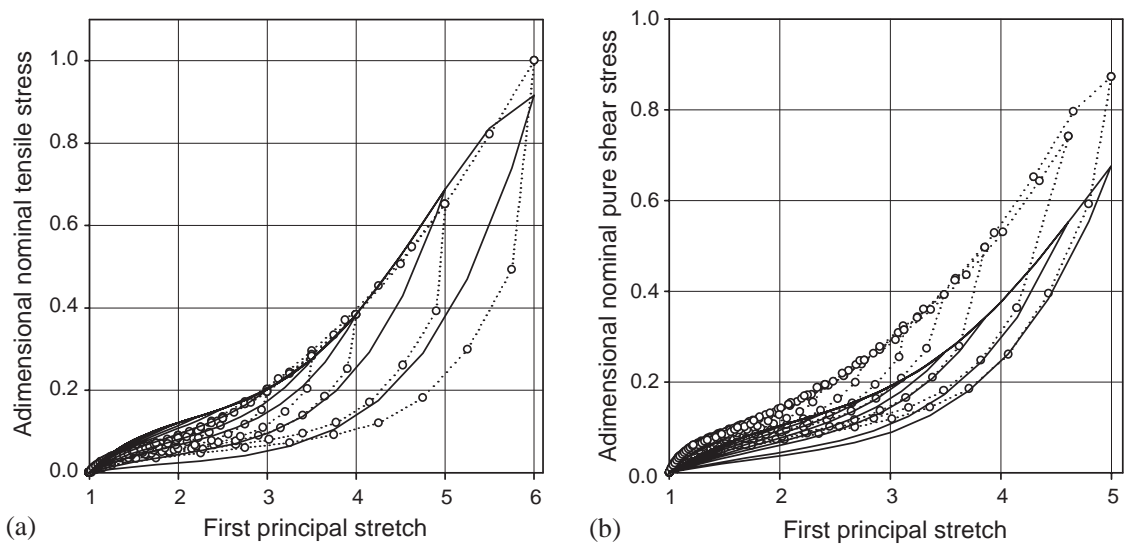

Fig. 7. Identification results, (a) uniaxial tensile and (b) pure shear: $(\cdots \circ \cdots)$ experiments and $(-)$ our hyperelastic model with damage.

\section{Numerical examples}

In order to demonstrate the efficiency of the present approach for engineering applications, the constitutive equation was implemented in the commercial software Abaqus (Hibbitt and Karlsson and Sorensen Inc., S., 1999) with the help of the UMAT facility. Both the Cauchy stress tensor and the fourth-order elasticity tensor are needed. An accurate derivation of this elasticity tensor is of major importance to ensure and accelerate convergence.

Both plane stress and three-dimensional formulations were implemented. The plane stress implementation is quite simple, because the numerical difficulty due to the incompressibility assumption is automatically satisfied through the change of thickness in the normal direction.

Two examples are described in the next paragraphs.

\subsection{Square perforated sheet}

The first example is a well-known plane stress hyperelasticity problem where an initial $20 \times 20 \mathrm{~mm}^{2}$ strip with a circular hole of diameter $10 \mathrm{~mm}$ is stretched in both horizontal and vertical directions. The corresponding initial mesh is shown in Fig. 8(a). This example will exhibit the strong dependence of stress-softening on the deformation history. Three different deformation paths are investigated (amplitudes of cycles are identical) and their corresponding damage distributions are presented in Fig. 8:

(i) the sheet is stretched to $185 \%$ in direction 2 then unloaded (Fig. $8(\mathrm{~b})$ ), $\max d=$ 0.33 ,

(ii) the sheet is stretched to $185 \%$ in direction 2 , unloaded, then stretched to $185 \%$ in direction 1 and finally unloaded (Fig. 8(c)), $\max d=0.33$, 
(a)
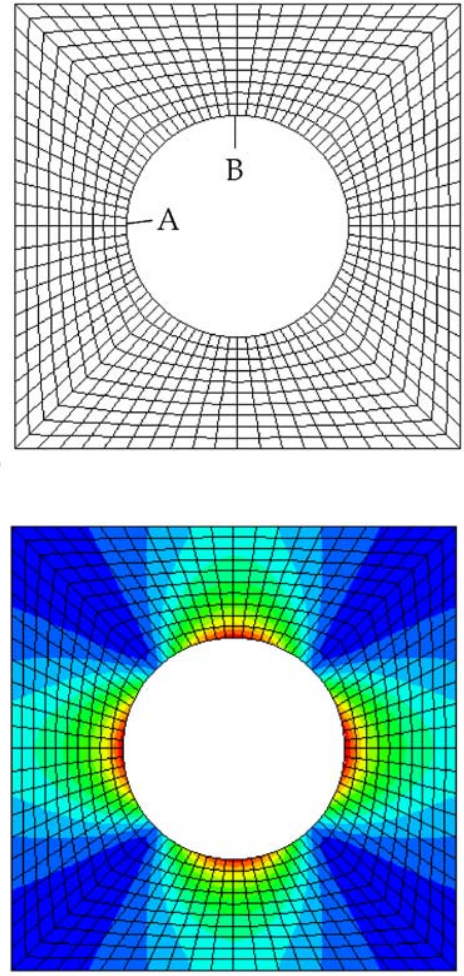

(c)

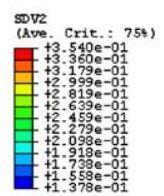

(b)
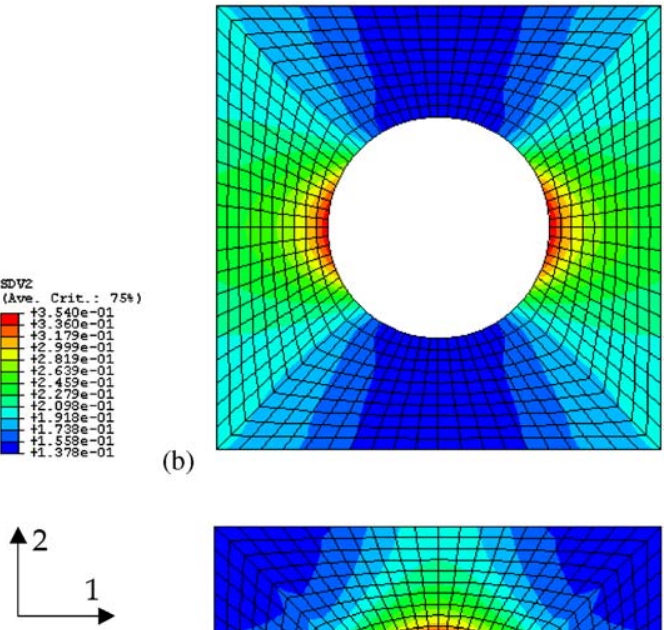

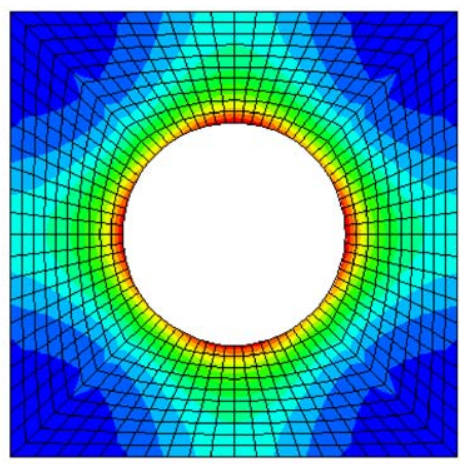

(d)

Fig. 8. Finite element simulation of a square perforated sheet: (a) undeformed mesh, (b) damage distribution after a cycle in dir. 2, (c) damage distribution after a cycle in dir. 2 followed by a cycle in dir. 1 and (d) damage distribution after a cycle in the diagonal direction.

(iii) the sheet is stretched to $185 \sqrt{2} \%$ in one of the diagonal directions then unloaded (Fig. 8(d)), $\max d=0.35$.

The influence of the loading history on the damage distribution can be highlighted by comparing results (ii) and (iii). The two samples were subjected to the same deformation state in both directions 1 and 2. Only loading paths differ. In case (ii), the sample is first stretched then relaxed in direction 2. This first cycle weakens the structure in the neighbourhood of the hole, in direction 1 (see Fig. 8(b)). Then, the second cycle applied in direction 1 modifies a new unloaded non-homogeneous damage distribution. So, the final damage distribution is non-symmetric as shown in Fig. 8(c). In the loading case (iii), both cycles (in directions 1 and 2) are applied simultaneously and the final damage distribution respects the structural symmetry as displayed in Fig. 8(d). In order to exhibit more precisely this phenomenon, consider Fig. 9 which presents the evolution of the damage state in elements denoted A and B in Fig. 8(a) for the two loading history (ii) and (iii). In this figure, it is clearly shown that case (ii) leads to a non-symmetric damage distribution. 


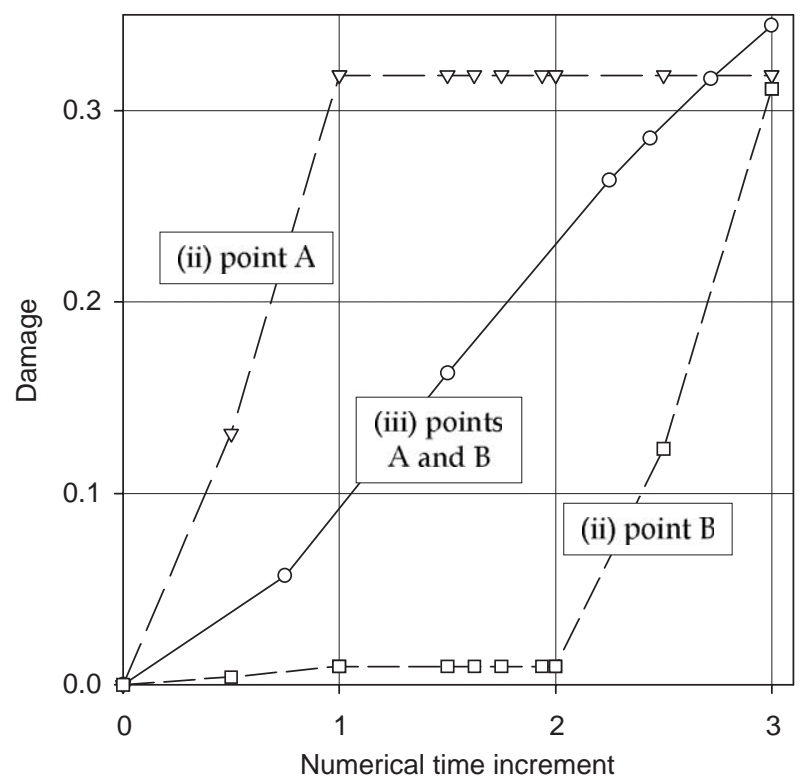

Fig. 9. Evolution of the damage in elements lying on the edge of the hole: $(--\nabla--)$ loading (ii) at point A, (- - $\square$ - -) loading (ii) at point B, (-o-) loading (iii) at points A and B.

\subsection{Simultaneous uniaxial tension and torsion of a diabolo sample}

The second example is a three-dimensional problem that highlights the capability of our approach to predict the behaviour of complex parts. The structure is a rubber diabolo sample. Its length and width are, respectively, 30 and $20 \mathrm{~mm}$. The sample is stretched in its length direction (uniaxial tension) and twisted by rotating one of its ends.

Fig. 10(a) presents an example of damage distribution in the part. The maximum of damage takes place in the central region of the sample because of its geometry. Indeed, this sample geometry is used to maximize strain and stress in the minimum section. In order to investigate the influence of loading conditions on damage, the sample is twisted for different levels of uniaxial tension. This tensile loading is performed by controlling the relative displacement of diabolo ends. The corresponding results are presented in Fig. 10(b), in which damage in the central region is drawn as a function of the torsional angle for different values of the relative displacement of ends. As exhibited above, under uniaxial tensile conditions, i.e. when the torsional angle is null, damage is an increasing function of the tension. Moreover, for a given value of pre-tension, damage increases as the torsional angle increases, because the deformation measure also increases. Nevertheless, for a torsional angle equal to $2 \pi$, the smaller is the pre-tension, the larger is the damage. This can be explained by the deformation measure adopted to drive the evolution of damage. In fact, this measure being a function of the first principal invariants of $\mathbf{B}$, it takes into account the whole deformation state that may 


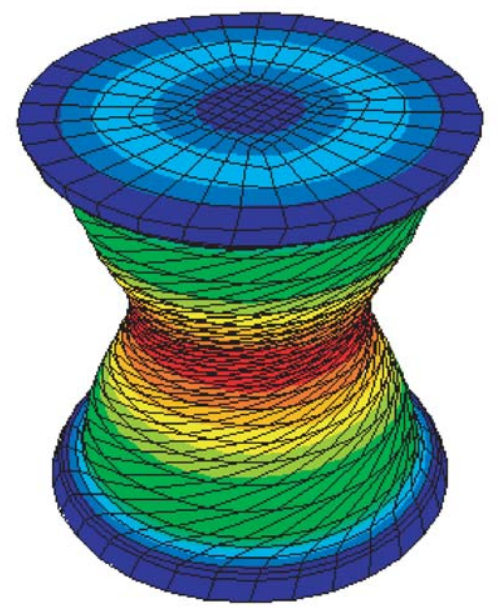

(a)

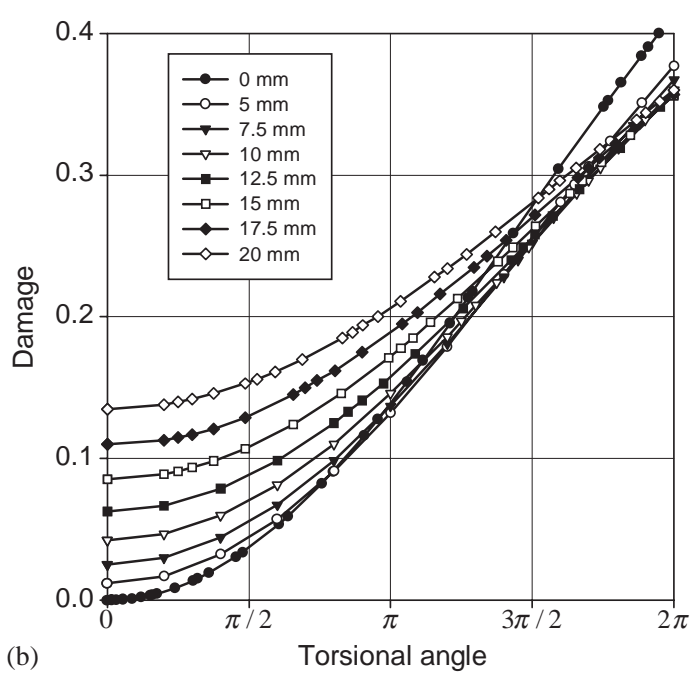

Fig. 10. (a) Damage distribution in the diabolo sample under simultaneous uniaxial tensile and torsional loading conditions. (b) Maximum damage vs torsional angle, influence of the tensile pre-loading.

be larger without pre-tension. Indeed, for large torsional angles, local shear stresses dominate and increase as pre-tension decreases, this phenomenon being rendered by the measure of deformation.

Finally, note that these samples will be used in further works to investigate crack initiation in elastomers under fatigue loading, more precisely to examine the influence of the deformation state on crack occurrence. As shown with these preliminary simulations, the Mullins effect should be considered in fatigue problems because stress-softening highly reduces local stiffness and will certainly influence the fatigue life of elastomers.

\section{Conclusion}

The present work demonstrates the ability and limitations of Continuum Damage Mechanics to describe the Mullins effect in elastomers. The material considered here is natural rubber; it is assumed non-linear elastic, isotropic and incompressible, viscous effects not being taken into account. The general framework of hyperelasticity with damage is derived. Restrictions of this theory are exhibited and it is shown that CDM should be employed with care to model stress-softening in elastomers. Moreover, the connection with two-network (Green and Tobolsky, 1946) or two-phase (Mullins and Tobin, 1957) theories is demonstrated; the properties of the deformation measure used in the evolution equation of the damage variable are established. Then, this evolution equation is constructed using both uniaxial tensile and simple shear experiments. The inapplicability of models of this kind in strain-hardening parts of the stress-strain curve is demonstrated. Nevertheless, the identification of material parameters leads to 
good qualitative results. From a numerical point of view, the model is implemented in Abaqus for both plane stress and three-dimensional cases. Convergence of the model is ensured even for very large strain. Some examples highlight the influence of loading paths on the response of the material, and demonstrate the importance of considering stress-softening for the development ofi ndustrial parts.

Finally, one last remark has to be made. Connections between the two phenomenological theories used for the Mullins effect, i.e. damage mechanics and two-network (or two-phase) approaches, are now established. Then, the only remaining difficulty is the determination of the evolution equation that drives the parameter used to correct the strain-energy function, whatever the terminology employed: damage parameter, network reformation function or transformation function between hard and soft regions of rubber. Here, we showed that the use of the maximum deformation endured previously by the material as the damage criterion is not sufficient to accurately describe strain-hardening. Thus, the evolution equation of the stress-softening variable should be also written in terms of the current deformation measure and not only in terms of the maximum deformation previously endured by the material. Such approaches were recently proposed byMiehe and Keck (2000) and El ías-Zúñiga and Beatty (2002).

\section{References}

Arruda, E.M., Boyce, M.C., 1993. A three dimensional constitutive model for the large stretch behavior of rubber elastic materials. J. Mech. Phys. Solids 41, 389-412.

Beatty, M.F., Krishnaswamy, S., 2000. A theory of stress-softening in incompressible isotropic materials. J. Mech. Phys. Solids 48, 1931-1965.

Bergström, J.S., Boyce, M.C., 1998. Constitutive modeling of the large stain time-dependent behavior of elastomers. J. Mech. Phys. Solids 46, 931-954.

Boyce, M.C., Arruda, E.M., 2000. Constitutive models of rubber elasticity: a review. Rubber Chem. Technol. 73, 505-523.

Bueche, F., 1960. Molecular basis for the Mullins effect. J. Appl. Polym. Sci. 4, 107-114.

Bueche, F., 1961. Mullins effect and rubber-filler interaction. J. Appl. Polym. Sci. 5, 271-281.

Charlton, D.J., Yang, J., Teh, K.K., 1994. A review of methods to characterize rubber elastic behavior for use in finite element analysis. Rubber Chem. Technol. 67, 481-503.

De Souza Neto, E.A., Peric, D., Owen, D.R.J., 1994. A phenomenological three-dimensional rate-independent continuum damage model for highly filled polymers: formulation and computational aspects. J. Mech. Phys. Solids 42, 1533-1550.

Drozdov, A.D., Dorfmann, A.I., 2001. Stress-strain relations in finite viscoelastoplasticity of rigid-rod networks: applications to the Mullins effect. Continuum Mech. Thermodyn. 13, 183-205.

El ías-Zúñiga, A., Beatty, M.F., 2002. A new phenomenological model for stress-softening in elastomers. Z. Angew. Math. Phys. 53, 794-814.

Gent, A.N., Thomas, A.G., 1958. Forms of the stored (strain) energy function for vulcanized rubber. J. Polym. Sci. 28, 625-637.

Govindjee, S., Simo, J.C., 1991. A micro-mechanically based continuum damage model for carbon black-filled rubbers incorporating Mullins' effect. J. Mech. Phys. Solids 39, 87-112.

Govindjee, S., Simo, J.C., 1992. Mullins effect and the strain amplitude dependence of the storage modulus. Int. J. Solids Struct. 29, 1737-1751.

Green, M.S., Tobolsky, A.V., 1946. A new approach for the theory of relaxing polymeric media. J. Chem. Phys. 14, 87-112.

Gurtin, M.E., Francis, E.C., 1981. Simple rate-independent model for damage. J. Spacecraft 18, $285-286$.

Hart-Smith, L.J., 1966. Elasticity parameters for finite deformations of rubber-like materials. Z. Angew. Math. Phys. 17, 608-626. 
Hibbitt and Karlsson and Sorensen Inc., S., 1999. ABAQUS/Standard User's Manual V5. 8.

Huntley, H.E., Wineman, A.S., Rajagopal, K.R., 1996. Chemorheological relaxation, residual stress and permanent set arising in radial deformation of an elastomeric hollow sphere. Math. Mech. Solids 1, 267-299.

Huntley, H.E., Wineman, A.S., Rajagopal, K.R., 1997. Stress softening, strain localization and permanent set in the circumferential shear of an incompressible elastomeric cylinder. IMA J. Appl. Math. 59, 309-338.

James, H.M., Guth, E., 1943. Theory of the elastic properties of rubber. J. Chem. Phys. 11, 455-481.

James, H.M., Guth, E., 1947. Theory of the increase in rigidity of rubber during cure. J. Chem. Phys. 15, 669-683.

Johnson, M.A., Beatty, M.F., 1993a. A constitutive equation for the Mullins effect in stress controlled extension experiments. Continuum Mech. Thermodyn. 5, 301-318.

Johnson, M.A., Beatty, M.F., 1993b. The Mullins effect in uniaxial extension and its influence on transverse vibration of rubber string. Continuum Mech. Thermodyn. 5, 83-115.

Johnson, M.A., Beatty, M.F., 1995. The Mullins effect in equibiaxial extension and its influence on the inflation of a balloon. Int. J. Eng. Sci. 33, 223-245.

Kachanov, L.M., 1958. Time of the rupture process under creep conditions. Izv. Akad. Nauk. S.S.R. Otd. Tech. Nauk. 8, 26-31.

Kuhn, W., Gr ün, F., 1942. Beziehungen zwichen elastischen Konstanten und Dehnungsdoppelbrechung hochelastischer Stoffe. Kolloideitschrift 101, 248-271.

Lemaitre, J., Chaboche, J.L., 1985. Mécanique des Matériaux Solides. Dunod, Paris.

Lion, A., 1996. A constitutive model for carbon black filled rubber: experimental investigations and mathematical representation. Continuum Mech. Thermodyn. 8, 153-169.

Marckmann, G., Verron, E., Gornet, L., Chagnon, G., Charrier, P., Fort, P., 2002. A theory of network alteration for the Mullins effect. J. Mech. Phys. Solids 50, 2011-2028.

Maugin, G.A., 1999. The Thermodynamics of Nonlinear Irreversible Behaviors: an Introduction. In: World Scientific Series on Nonlinear Science, Series A, Vol. 27. World Scientific, Singapore.

Miehe, C., 1995. Discontinuous and continuous damage evolution in Ogden-type large-strain elastic materials. Eur. J. Mech. A/Solids 14, 697-720.

Miehe, C., Keck, J., 2000. Superimposed finite elastic-viscoelastic-plastoelastic stress response with damage in filled rubbery polymers. Experiments, modelling and algorithmic implementation. J. Mech. Phys. Solids $48,323-365$.

Mooney, M., 1940. A theory ofl arge elastic deformation. J. Appl. Phys. 11, 582-592.

Mullins, L., 1969. Softening of rubber by deformation. Rubber Chem. Technol. 42, 339-362.

Mullins, L., Tobin, N.R., 1957. Theoretical model for the elastic behavior of filler-reinforced vulcanized rubbers. Rubber Chem. Technol. 30, 551-571.

Ogden, R.W., 1972. Large deformation isotropic elasticity-on the correlation of theory and experiment for incompressible rubberlike solids. Proc. Roy. Soc. London A 326, 565-584.

Ogden, R.W., 1984. Non-Linear Elastic Deformations. Ellis Horwood, Chichester.

Ogden, R.W., Roxburgh, D.G., 1999. A pseudo-elastic model for the Mullins effect in filled rubber. Proc. Roy. Soc. London A 455, 2861-2877.

Rajagopal, K.R., Wineman, A.S., 1992. A constitutive equation for nonlinear solids which undergo deformation induced by microstructural changes. Int. J. Plast. 8, 385-395.

Rivlin, R.S., 1948. Large elastic deformation ofi sotropic materials-IV. Further developments of the general theory. Philos. Trans. Roy. Soc. A 241, 379-397.

Rivlin, R.S., Saunders, D.W., 1951. Large elastic deformations ofi sotropic materials-vii. experiments on the deformation of rubber. Philos. Trans. Roy. Soc. A 243, 251-288.

Simo, J.C., 1987. On a fully three-dimensional finite-strain viscoelastic damage model: formulation and computational aspects. Comput. Methods Appl. Mech. Eng. 60, 153-173.

Simo, J.C., Hughes, T.J.R., 1998. Computational Inelasticity. Springer, New York.

Treloar, L.R.G., 1944. Stress-strain data for vulcanised rubber under various types of deformation. Trans. Faraday Soc. 40, 59-70.

Treloar, L.R.G., Riding, G., 1979. A Non-Gaussian theory for rubber in biaxial strain. I. Mechanical properties. Proc. Roy. Soc. London A 369, 261-280. 
Truesdell, C., Noll, W., 1965. Handbuch der Physik Bd, III/3, The Nonlinear Field Theories of Mechanics. Springer, Berlin.

Voyiadjis, G.Z., Ju, J.W., Chaboche, J.L. (Eds.), 1998. Damage Mechanics in Engineering Materials, Studies in Applied Mechanics, Vol. 46. Elsevier, Amsterdam.

Wineman, A.S., Huntley, H.E., 1994. Numerical simulation of the effect of damaged induced softening on the inflation of a circular rubber membrane. Int. J. Solids Struct. 31, 3295-3313.

Wineman, A.S., Rajagopal, K.R., 1990. On a constitutive theory for materials undergoing microstructural changes. Arch. Mech. 42, 53-75.

Wu, P.D., van der Giessen, E., 1993. On improved network models for rubber elasticity and their applications to orientation hardening in glassy polymers. J. Mech. Phys. Solids 41, 427-456.

Yeoh, O.H., 1990. Characterization of elastic properties of carbon-black-filled rubber vulcanizates. Rubber Chem. Technol. 63, 792-805.

Yeoh, O.H., 1993. Some forms of the strain energy function for rubber. Rubber Chem. Technol. 66, 754-771. 